\title{
ON THE DIFFERENTIAL SYSTEM GOVERNING FLOWS IN MAGNETIC FIELD WITH DATA IN $L^{p}$
}

\author{
FENGXIN CHEN \\ Department of Mathematics \\ Brigham Young University \\ Provo, Utah 84602, USA \\ PING WANG \\ Department of Mathematics \\ Penn State University \\ Schuylkill Haven, PA 17972, USA \\ CHAOSHUN QU \\ Department of Mathematics \\ Yunnan University, P.R. CHINA
}

(Received March 25, 1996 and in revised form May 13, 1996)

ABSTRACT. In this paper we study the system governing flows in the magnetic field within the earth. The system is similar to the magnetohydrodynamic (MHD) equations. For initial data in space $L^{p}$, we obtained the local in time existence and uniqueness of weak solutions of the system subject to appropriate initial and boundary conditions.

KEY WORDS AND PHRASES: Fluid, magnetic field, work solution.

1991 AMS SUBJECT CLASSIFICATION CODES: 35K55, 35Q35, 76D9.

\section{INTRODUCTION}

We consider in this work the following differential system arising from geophysics (cf. Hide [1]), which governs the flow of an electrically-conducting fluid in the presence of a magnetic field, when referred to a frame which rotates with angular velocity $\Omega$ relative to an inertial frame:

$$
\begin{gathered}
\frac{\partial v}{\partial t}+(v \cdot \nabla) v=\nu \Delta v-\frac{1}{\rho} \nabla p-2 \Omega \times v+\frac{1}{\rho \mu}(\nabla \times b) \times b+f(x), \\
\frac{\partial b}{\partial t}=\lambda \Delta b+\nabla \times(v \times b)-\frac{1}{\mu} \nabla q+g(x), \\
\operatorname{div} v=0 ; \quad \operatorname{div} b=0,
\end{gathered}
$$

where $v$ is the Eulerian flow velocity, $\rho$ is the density, $b$ is the magnetic field, $p$ is the pressure, $\nu, \mu$ are respectively constants of kinematical viscosity, magnetic permeability, $\lambda=\frac{\eta}{\mu}$ with electrical resistivity $\eta$, and $f(x), g(x)$ are volume forces. Let $K$ be an open bounded subset of $R^{n}$ with boundary $\Gamma$. The initial and boundary conditions are as follows respectively:

$$
\begin{gathered}
v=0 ; \quad b=0 \text { on } \Gamma \\
v(x, 0)=v_{0} ; \quad b(x, 0)=b_{0} \text { for } x \in K
\end{gathered}
$$

where $n$ is the outward unit normal on $\Gamma$.

The existence of solutions of systems (1.1)-(1.5) in $L^{2}$ has been proved in Qu et al. [2]. Some regularity properties and large time behaviors of the solutions for a similar system, the MHD equations, are obtained in Sermange [3] and Temam [4]. 
In this work we consider the initial value problem for the above system in infinite cylinder $S_{T}=(0, T) \times R^{n}$ with initial data $v_{0}, b_{0} \in L^{p}$. Following Fabes et al. [5], we consider the solution of (1.1)-(1.5) in weak form. And we prove the local existence and uniqueness of weak solution of the system in $L^{p}$ space.

This article is arranged in the following order. In Section 2, we first introduce some notations and definitions and formulate the setting of our problem. Then we prove that solving the system (1.1)-(1.5) is equivalent to solving a nonlinear integral equation. Applying a well-known imbedding theorem, we prove in Section 3 the uniqueness of the solution for all $T$ and the existence of the solution for small value of $T$.

\section{NOTATIONS AND AN INTEGRAL EQUATION}

In this section, we introduce some notations and define the weak solution of differential system (1.1)-(1.5). Then reduce the system to an integral equation. We prove later in this section that the differential system (1.1)-(1.5) and the integral equation are equivalent.

We use $L^{p, q}$ to denote the standard functional space consisting of Lebesque measurable vector functions $f=\left(f_{1}, f_{2}, \cdots, f_{n}\right)$ with the following property

$$
\|f\|_{p, q}=\sum_{j=1}^{n}\left[\int_{0}^{T}\left(\int_{R^{n}}\left|f_{\jmath}(x, t)\right|^{p} d x\right)^{\frac{q}{p}}\right]^{\frac{1}{q}}<\infty .
$$

Denote $\mathcal{L}^{p, q}\left(S_{T}\right)=L^{p, q}\left(S_{T}\right) \times L^{p, q}\left(S_{T}\right)$ with the standard product norm $\|(v, b)\|_{p, q}=\|v\|_{p, q}+\|b\|_{p, q}$ and $L^{p}\left(R^{n}\right)=L^{p}\left(R^{n}\right) \times \cdots \times L^{p}\left(R^{n}\right)$ with the norm $\|g\|_{p}=\sum_{z=1}^{n}\left\|g_{\imath}\right\|_{p}$ for $g \in L^{p}\left(R^{n}\right)$.

Let $\mathcal{S}\left(R^{n}\right)$ denote the space of rapidly decreasing functions on $R^{n}, \mathcal{S}^{\prime}\left(R^{n}\right)$ the space of temperated distributions, and $\mathcal{D}_{T}$ the space of functions $\phi(x, t)=\left(\phi_{1}(x, t), \cdots, \phi_{n}(x, t)\right)$ with the properties: $\phi_{i} \in \mathcal{S}\left(R^{n+1}\right), \phi_{i}(x, t)=0$ for $t \geq T ; \operatorname{div} \phi=\sum_{i=1}^{n} D_{x_{2}} \phi_{i}(x, t)=0$ for all $t$.

DEFINITION 2.1. A function $u=(v, b)$ is a weak solution of (1.1)-(1.5) with intial divergence free data $\left(v_{0}, b_{0}\right) \in L^{p}\left(R^{n}\right) \times L^{p}\left(R^{n}\right)$ if the following conditions hold:

(1) $u(x, t) \in \mathcal{L}^{p, q}\left(S_{T}\right)$ for some $p, q$ with $p, q>2$;

(2) For $\phi, \psi \in \mathcal{D}_{T}$,

$$
\begin{aligned}
& \int_{0}^{T} \int_{R^{n}}\left\langle v,\left(\nu \Delta+D_{t}\right) \phi\right\rangle d x d t+\int_{0}^{T} \int_{R^{n}}\langle b,(\nabla \phi) b\rangle d x d t \\
& \quad+\int_{0}^{T} \int_{R^{n}}\langle v, 2 \Omega \times \phi\rangle d x d t-\frac{1}{\rho \mu} \int_{0}^{T} \int_{R^{n}}\langle b,(\nabla \phi) b\rangle d x d t \\
& =-\int_{R^{n}}\left\langle v_{0}, \phi(x, 0)\right\rangle d x+\int_{0}^{T} \int_{R^{n}}\langle f(x, t), \phi\rangle d x d t ; \\
& \int_{0}^{T} \int_{R^{n}}\left\langle b,\left(\lambda \Delta+D_{t}\right) \psi\right\rangle d x d t+\int_{0}^{T} \int_{R^{n}}\langle v,(\nabla \psi) b\rangle d x d t \\
& \quad-\int_{0}^{T} \int_{R^{n}}\langle b,(\nabla \psi) v\rangle d x d t \\
& =-\int_{R^{n}}\left\langle b_{0}, \psi(x, 0)\right\rangle d x+\int_{0}^{T} \int_{R^{n}}\langle g(x, t), \psi\rangle d x d t ;
\end{aligned}
$$

(3) For almost every $t \in[0, T], \operatorname{div} v(x, t)=\operatorname{div} b(x, t)=0$ in the distributional sense.

Following Fabes et al. [5], we can find a divergence free matrix fundamental solution $E_{\imath, \mathrm{J}}$ for $n$ dimensional heat equation. We define matrices $\left(E_{\imath, j}^{k}\right), k=1,2$ as follows:

$$
E_{i, j}^{k}=\delta_{i, j} \Gamma_{k}(x, t)-R_{i} R_{j} \Gamma_{k}(x, t),
$$

where 


$$
\Gamma_{1}=\frac{e^{-\frac{|x|^{2}}{4 t}}}{(4 \pi \nu t)^{\frac{n}{2}}}, \Gamma_{2}=\frac{e^{-\frac{|x|^{2}}{4 \lambda t}}}{(4 \pi \lambda t)^{\frac{n}{2}}}
$$

$R$, is the $j$ th Riesz transform, namely $R$, is a singular integral operator on $L^{p}\left(R^{n}\right), 1<p<\infty$, defined as

$$
R_{j}(f)=\text { P.V. } C_{\jmath} \int_{R^{n}}\left(x_{\jmath}-y_{\jmath}\right)|x-y|^{-n-1} f(y) d y .
$$

It is easy to check that $E^{k}=\left(E_{\imath, j}^{k}\right), k=1,2$, are symmetric and divergence free, i.e.,

$$
\sum_{j=1}^{n} D_{\jmath} E_{i, j}^{k}(x, t)=0, t>0 .
$$

In the formulas above we assumed that classical derivatives were taken for $t>0$; distributional derivatives were taken for the limit as $t \rightarrow 0$.

It is easy to see that if $\operatorname{div} f=0$, as $t \rightarrow 0$,

$$
\sum_{j} \int_{R^{n}} E_{z, j}^{k} f_{j}(y-x) d x \rightarrow f_{i}(y) \text { in } L^{p}, 1<p<\infty .
$$

Now we define an integral operator $A(v, w)$ for $v=\left(v_{1}, \cdots, v_{n}\right), w=\left(w_{1}, \cdots, w_{n}\right)$. Denote

$$
\begin{gathered}
B_{k}(v, w)(x, t)=\int_{0}^{t} \int_{R^{n}}\left\langle v(y, s), \nabla E^{k}(x-y, t-s)\right\rangle w(y, s) d y d s ; \\
D(v)(x, t)=\int_{0}^{t} \int_{R^{n}}\left\langle v(y, s), 2 \omega \times E^{1}(x-y, t-s)\right\rangle d y d s .
\end{gathered}
$$

For $u_{1}=\left(v_{1}, b_{1}\right), u_{2}=\left(v_{2}, b_{2}\right)$, let

$$
A\left(u_{1}, u_{2}\right)=\left(\begin{array}{l}
B_{1}\left(v_{1}, v_{2}\right)-\frac{1}{\rho \mu} B_{1}\left(b_{1}, b_{2}\right) \\
\frac{1}{2}\left[B_{2}\left(v_{1}, b_{1}\right)-B_{2}\left(b_{1}, v_{1}\right)+B_{2}\left(v_{2}, b_{2}\right)-B_{2}\left(b_{2}, v_{2}\right)\right]
\end{array}\right) .
$$

Consider the following integral equation

$$
u+A(u, u)+D(u)=u^{0}+f^{0},
$$

where

$$
u^{0}=\left(\begin{array}{l}
\int_{R^{n}} \Gamma_{1}(x-y, t) v_{0}(y) d y \\
\int_{R^{n}}^{n} \Gamma_{2}(x-y, t) b_{0}(y) d y
\end{array}\right)
$$

and

$$
f^{0}=\left(\begin{array}{c}
\int_{0}^{t} \int_{R^{n}} E^{1}(x-y, t-s) f(y, s) d y d s \\
\int_{0}^{t} \int_{R^{n}} E^{2}(x-y, t-s) g(y, s) d y d s
\end{array}\right) .
$$

We are ready to state the main result in this section.

THEOREM 2.1. Let $v_{0}, b_{0} \in L^{r}, 1 \leq r<\infty$, be divergence free weakly. $u(x, t)=(v(x, t), b(x, t)) \in$ $\mathcal{L}^{p, q}\left(S_{T}\right), p, q \geq 2, p<\infty$, is a weak solution of (1.1)-(1.5) with intial value $\left(v_{0}, b_{0}\right)$ if and only if $u$ is a solution of integral equation (2.10).

PROOF. We prove the theorem for the case of $f=g=0$ (the proof for the case when $f \neq 0, g \neq 0$ is similar).

Let us first assume that $u=(v, b)$ is a solution of the integral equation (2.10). Set $v_{1}=B_{1}(v, v), v_{2}=B_{1}(b, b), b_{1}=B_{2}(v, b), b_{2}=B_{2}(b, v)$. Following the argument in the proof of 
Theorem 2.1 in Fabes et al. [5], we can prove that $v_{1}, v_{2}, b_{1}, b_{2}$ are all weakly divergence free for almost every $t \in[0, T]$. Since $\left\{E_{i j}^{k}\right\}$ is weakly divergence free by $(2.5)$, it can be easily verified that $D(u)$ is also divergence free. Hence, both $v=v^{0}-v_{1}+\frac{1}{\mu \rho}+D(u), b=b^{0}-b_{1}+b_{2}$ are divergence free.

To see $u=(v, b)$ satisfies (1.1), (1.2), we first show that for $\phi \in \mathcal{D}_{T}, v, w \in \mathcal{L}^{p, q}\left(S_{T}\right)$ with $\operatorname{div} v=\operatorname{div} w=0$,

$$
\begin{aligned}
& \int_{0}^{T} \int_{R^{n}}\left\langle B_{1}(v, w), D_{T} \phi+\nu \Delta \phi\right\rangle d x d t=\int_{0}^{T} \int_{R^{n}}\langle v, \nabla \phi(w)\rangle d x d t \\
& \int_{0}^{T} \int_{R^{n}}\left\langle B_{2}(v, w), D_{T} \phi+\lambda \Delta \phi\right\rangle d x d t=\int_{0}^{T} \int_{R^{n}}\langle v, \nabla \phi(w)\rangle d x d t
\end{aligned}
$$

In fact,

$$
\begin{aligned}
B_{1}(v, w)(x, t)= & \int_{0}^{t} \int_{R^{n}}\left\langle v(y, s), \nabla E^{1}(x-y, t-s)\right\rangle w(y, s) d y d s \\
= & \int_{0}^{t} \int_{R^{n}} \Gamma_{1}(x-y, t-s)(\nabla v(w))(y, s) d y d s \\
& \quad-\int_{0}^{t} \int_{R^{n}} \Gamma_{1}(x-y, t-s)\left(R_{1} R_{j}\right)(\nabla v(w))(y, s) d y d s .
\end{aligned}
$$

This implies that

$$
\left(\nu \Delta-D_{t}\right) B_{1}(v, w)=-\nabla v(w)+R_{i} R_{\jmath}(\nabla v(w)) .
$$

Since $\left(R_{i} R_{\jmath}\right)(\phi)=0$ for $\phi \in \mathcal{D}_{T}$, we obtain

$$
\int_{0}^{T} \int_{R^{n}}\left\langle B_{1}(v, w), D_{T} \phi+\nu \Delta \phi\right\rangle d x d t=\int_{0}^{T} \int_{R^{n}}\langle v, \nabla \phi(w)\rangle d x d t
$$

Similarly, we can prove (2.14) and

$$
\int_{0}^{T} \int_{R^{n}}\left\langle v, D_{T} \phi+\nu \Delta \phi\right\rangle d x d t=\int_{0}^{T} \int_{R^{n}}\langle 2 \Omega \times v, \phi\rangle d x d t .
$$

Considering (2.13), (2.14), (2.16) and the fact that $u=(v, b)$ is a solution of the integral equation, we have

$$
\begin{aligned}
& \int_{0}^{T} \int_{R^{n}}\left\langle v,\left(\nu \Delta+D_{t}\right) \phi\right\rangle d x d t+\int_{0}^{T} \int_{R^{n}}\langle b,(\nabla \phi) b\rangle d x d t \\
& \quad+\int_{0}^{T} \int_{R^{n}}\langle v, 2 \Omega \times \phi\rangle d x d t-\frac{1}{\rho \mu} \int_{0}^{T} \int_{R^{n}}\langle b,(\nabla \phi) b\rangle d x d t \\
& =\int_{0}^{T} \int_{R^{n}}\left\langle v_{0}-B_{1}(v, v)+\frac{1}{\rho \mu} B_{1}(b, b)-D(v), D_{t} \phi+\nu \Delta \phi\right\rangle d x d t \\
& \quad+\int_{0}^{T} \int_{R^{n}}\langle v, \nabla \phi(v)\rangle d x d t-\int_{0}^{T} \int_{R^{n}}\langle v, 2 \Omega \times \phi\rangle d x d t-\frac{1}{\rho \mu} \int_{0}^{T} \int_{R^{n}}\langle b, \nabla \phi(b)\rangle d x d t \\
& =\int_{0}^{T} \int_{R^{n}}\left\langle v^{0}, D_{t} \phi+\nu \Delta \phi\right\rangle d x d t \\
& =-\int_{R^{n}}\left\langle v_{0}, \phi(x, 0)\right\rangle d x .
\end{aligned}
$$

since $\langle\Omega \times v, \phi\rangle=-\langle v, \Omega \times \phi\rangle$. Similarly we can show that (2.2) in Definition 2.1 holds. Hence, $u$ is a weak solution of (1.1)-(1.5).

On the other hand, suppose $u$ is a weak solution of (1.1)-(1.5) with initial data $\left(v_{0}, b_{0}\right)$. Choose $a \in C^{\infty}\left(R^{n}\right)$ such that $a(x)=1$ when $|x| \geq 2 ; a(x)=0$ when $|x| \leq 1$ and choose $\psi \in C^{\infty}\left(R^{n}\right)$ such that $\psi(t)=1$ when $|t| \geq 2 ; \psi(t)=0$ when $|t| \leq 1$. Let $E_{\imath}^{k}(\lambda, x, t)=\left\{E_{i, j}^{k}(\lambda, x, t)\right\}_{\jmath=1}^{k}$, 
$E_{\imath, j}^{k}(\lambda, x, t)=\mathcal{F}^{-1}\left(a_{\lambda}\left(E_{\imath, j}\right)\right)$, where $a_{\lambda}(x)=a(\lambda x), \mathcal{F}$ is the Fourier transform taken in $x$ variables. For $\psi_{\epsilon}(t)=\psi\left(\frac{t}{\epsilon}\right)$ and fixed $x, t$, we use $\phi_{2, \lambda}^{k}(y, s)=\psi(s+2) \psi_{\epsilon}(t-\epsilon) E_{i}^{k}(\lambda, x-y, t-s)$ as test functions in Definition 2.1 and following Fabes et al. [5] we can show that $u$ is a solution of (2.10).

\section{ON THE INTEGRAL EQUATION}

In this section we study the integral equation (2.10). We will show that it has a unique solution in $\mathcal{L}^{p, q}\left(S_{T}\right)$ with initial data in $\mathcal{L}^{p, q}\left(S_{T}\right)$. In the following, it is assumed that $F=0$ (the case $F \neq 0$ can be treated similarly).

Consider $A\left(u_{1}, u_{2}\right), D(u)$ as mappings from $\mathcal{L}^{p, q}\left(S_{T}\right)$ to $\mathcal{L}^{p, q}\left(S_{T}\right)$, when $\frac{n}{p}+\frac{2}{q} \leq 1$. The following theorem establishes the continuity of $A\left(u_{1}, u_{2}\right), D(u)$.

THEOREM 3.1. For $u_{1}, u_{2} \in \mathcal{L}^{p, q}\left(S_{T}\right)$, we have the following estimates:

(1) If $\frac{n}{p}+\frac{2}{q}=1$ with $n<p<\infty$, then

$$
\begin{gathered}
\left\|A\left(u_{1}, u_{2}\right)\right\|_{\mathcal{L}^{p, q}\left(S_{T}\right)} \leq C(n, p, q)\left\|u_{1}\right\|_{\mathcal{L}^{p, q}\left(S_{T}\right)}\left\|u_{2}\right\|_{\mathcal{L}^{p, q}\left(S_{T}\right)} \\
\left\|D\left(u_{1}\right)\right\|_{\mathcal{L}^{p, q}\left(S_{T}\right)} \leq C(n, p, q)\left\|u_{1}\right\|_{\mathcal{L}^{p, q}\left(S_{T}\right)} .
\end{gathered}
$$

(2) If $\frac{n}{p}+\frac{2}{q}<1$ with $n<p \leq \infty$, then

$$
\begin{aligned}
\left\|A\left(u_{1}, u_{2}\right)\right\|_{\mathcal{L}^{p, q}\left(S_{T}\right)} & \leq C(n, p, q) T^{\frac{1}{2}\left(1-\frac{n}{p}-\frac{2}{q}\right)}\left\|u_{1}\right\|_{\mathcal{L}^{p, q\left(S_{T}\right)}}\left\|u_{2}\right\|_{\mathcal{L}^{p, q}\left(S_{T}\right)} ; \\
\left\|D\left(u_{1}\right)\right\|_{\mathcal{L}^{p, q}\left(S_{T}\right)} & \leq C(n, p, q)\left\|u_{1}\right\|_{\mathcal{L}^{p, q}\left(S_{T}\right)} .
\end{aligned}
$$

PROOF. First of all, it is easy to show that

$$
\left|D_{x x} E_{z, j}^{k}(x, t)\right| \leq \frac{C}{\left(|x|+t^{\frac{1}{2}}\right)^{n+1}}
$$

for each $i, j$, . Therefore, for $a, b \in L^{p, q}\left(S_{T}\right)$,

$$
\left|B_{k}(a, b)\right| \leq C \int_{0}^{t} \int_{R^{n}} \frac{1}{\left(|x|+t^{\frac{1}{2}}\right)^{n+1}}|a(y, s)||b(y, s)| d y d s .
$$

To prove (3.1) and (3.2), we see for $\theta, 0<\theta<1$,

$$
\frac{1}{\left(|x|+t^{\frac{1}{2}}\right)^{n+1}} \leq \frac{C(\theta)}{|x|^{\theta(n+1)}} t^{\frac{(1-\theta)(n+1)}{2}} .
$$

Since $|a(y, s)|,|b(y, s)| \in L^{\frac{p}{2}}\left(R^{n}\right)$, if we choose $0<\theta<\frac{n}{n+1}, \frac{1}{p}=\frac{2}{p}-\frac{(n+1) \theta}{n}$, by Hardy-LittlewoodSobolev imbedding theorem,

$$
\left\|B_{k}(a, b)\right\|_{L^{p}\left(R^{n}\right)} \leq C(\theta, p, n) \int_{0}^{t} \frac{1}{(t-s)^{\frac{(n+1)(1-\theta)}{2}}}\||a(\cdot, s)| \cdot|b(\cdot, s)|\|_{L^{p}\left(R^{n}\right)} d s .
$$

If $\frac{n}{p}+\frac{2}{q}=1$ and $p>n$, then $\frac{1}{q}=\frac{2}{q}-\left[1-\frac{(n+1)(1-\theta)}{2}\right]$. Applying Hardy-Littlewood-Sobolev theorem again, we obtain

$$
\left\|B_{k}(a, b)\right\|_{L^{p}\left(R^{n}\right)} \leq C(n, p, q)\|a\|_{L^{p, q}\left(S_{T}\right)}\|b\|_{L^{p, q}\left(S_{T}\right)} .
$$

By the definition of $A\left(u_{1}, u_{2}\right)$, we see that (3.1) holds.

As for (3.2), we have 


$$
\begin{aligned}
D\left(u_{1}\right) & =\int_{0}^{t} \int_{R^{n}}\left\langle u_{1}(y, s), 2 \Omega \times E_{\imath, \jmath}^{1}(x-y, t-s)\right\rangle d y d s \\
& =\int_{0}^{t} \int_{R^{n}} \Gamma_{1}(x-y, t-s)\left(2 \Omega \times u_{1}+R_{\imath} R_{j}\left(2 \Omega \times u_{1}\right)\right)(y, s) d y d s .
\end{aligned}
$$

Since Riesz transform is continuous from $L^{p}$ to $L^{p}$ for $p>1, R_{2} R_{j}\left(2 \Omega \times u_{1}\right) \in \mathcal{L}^{p, q}\left(S_{T}\right)$ and

$$
\left\|R_{i} R_{j}(2 \Omega \times u)\right\|_{\mathcal{L}^{p, q\left(S_{T}\right)}} \leq C\|u\|_{\mathcal{L}^{\mathcal{P}, q}\left(S_{T}\right)}
$$

So from (3.89),

$$
\begin{aligned}
\|D(u)\|_{\mathcal{L}^{p, q}} & \leq \int_{0}^{t}\left\|\int_{R^{n}} \Gamma_{1}(x-y, t-s)\left(2 \Omega \times u+R_{\imath} R_{j}(2 \Omega \times u)\right)(y, s) d y\right\|_{\mathcal{L}_{\mathcal{P}, q\left(S_{T}\right)} d s} d s \\
& =C \int_{0}^{t}\left(\|2 \Omega \times u\|_{\mathcal{P}^{p, q\left(S_{T}\right)}}+\left\|R_{\imath} R_{j}(2 \Omega \times u)\right\|_{\left.\mathcal{L}_{\mathcal{P}, q\left(S_{T}\right)}\right) d s}\right. \\
& \leq C \int_{0}^{t}\|u(\cdot, s)\|_{\mathcal{C}^{p, q\left(S_{T}\right) d s}}
\end{aligned}
$$

from which (3.2) follows.

For (3.3), (3.4), we consider two cases: $p=\infty$ and $n<p<\infty$.

In the first case, for $a, b \in L^{p, q}\left(S_{T}\right)$,

$$
\left\|B_{k}(a, b)\right\|_{L^{\infty}\left(R^{n}\right)} \leq C \int_{0}^{t} \frac{1}{(t-s)^{\frac{1}{2}}}\|a(\cdot, s)\|_{L^{\infty}\left(R^{n}\right)}\|b(\cdot, s)\|_{L^{\infty}\left(R^{n}\right)} d s .
$$

If $q=\infty$, then

$$
\left\|B_{k}(a, b)\right\|_{L^{\infty}\left(R^{n}\right)} \leq C(T)^{\frac{1}{2}}\|a\|_{L^{\infty}\left(S_{T}\right)}\|b\|_{L^{\infty}\left(S_{T}\right)} .
$$

If $q<\infty$, by Hardy-Littlewood-Sobolev theorem, $\left\|B_{k}(a, b)(\cdot, t)\right\|_{L^{\infty}\left(R^{n}\right)} \in L^{r}(0, T)$, where $\frac{1}{r}=\frac{1}{q}-\frac{1}{2}$. Since $r>q$,

$$
\left\|B_{k}(a, b)\right\|_{L^{\infty, q}\left(S_{T}\right)} \leq C T^{\frac{1}{q}-\frac{1}{r}}\left\|B_{k}(a, b)\right\|_{L^{\infty, r}\left(S_{T}\right)} \leq C T^{\frac{1}{2}-\frac{1}{q}}\|a\|_{L^{\infty, q}\left(S_{T}\right)}\|b\|_{L^{\infty, q}\left(S_{T}\right)} .
$$

Finally, for the second case, $n<p<\infty$, we choose $q *$ and $r$ such that $q * \leq q \leq r$ and $\frac{1}{r}=\frac{2}{q^{*}}-\left[1-\frac{(n+1)(1-\theta)}{2}\right]$, where $\theta$ is chosen so that $\frac{1}{p}=\frac{2}{p}-\left[\frac{n-(n+1) \theta}{n}\right], 0<\theta<\frac{n}{n+1}$. Therefore, by Hardy-Littlewod-Sobolev theorem and Hölder's inequality, we have

$$
\begin{aligned}
\left\|B_{k}(a, b)\right\|_{L^{p, q}\left(S_{T}\right)} & \leq C T^{\frac{1}{q}-\frac{1}{r}}\left\|B_{k}(a, b)\right\|_{L^{p, r}\left(S_{T}\right)} \\
& \leq C T^{\frac{1}{q}-\frac{1}{r}}\|a\|_{L^{p, q}\left(S_{T}\right)}\|b\|_{L^{p, q}\left(S_{T}\right)} \\
& \leq C T^{\frac{1}{2}\left(1-\frac{n}{p}-\frac{2}{q}\right)}\|a\|_{L^{p, q}\left(S_{T}\right)}\|b\|_{L^{p, q(}\left(S_{T}\right)} .
\end{aligned}
$$

Hence using the same argument in the proof of (3.1), (3.2), we can similarly derive (3.3), (3.4).

Before stating the main results of this article, we introduce the following lemma Calderón [6]:

LEMMA 3.1. Let $T(u, v)=A(u, v)+l(u)+F$ be an operator on Banach space $E$, where $A(u, v)$ is bilinear, $l(u)$ is linear. The norm of the space is denoted by $\|\cdot\|$. If $T(u, v)$ satisfies

$$
\|T(u, v)\| \leq c_{1}\|u\|\|v\|+c_{2}\|u\|+\|F\|
$$

then $T(u, u)$ maps the ball $\mathcal{B}=\left\{\|u\| \leq s_{1}\right\}$ into itself if $s_{1}$ is the smaller root of

$$
c_{1} s^{2}+\left(c_{2}-1\right) s+\|F\|=0
$$

for $c_{1}, c_{2},\|F\|$ satisfying $\left(1-c_{2}\right)^{2}>4 c_{1}\|F\|, c_{1}<0,0 \leq c_{2}<1$. Moreover, $T(u, v)$ is a contraction mapping in ball $\mathcal{B}$ if $c_{1}, c_{2},\|F\|$ satisfy

$$
2 c_{1}\|F\|\left(\left(1-c_{2}\right)^{2}-4 c_{1}\|F\|\right)^{\frac{1}{2}}+c_{2}<1
$$


In the following, we use the above lemma to prove the existence of solutions of the integral equations (2.10).

THEOREM 3.2 (i). Suppose $\frac{n}{p}+\frac{2}{q}=1$ with $n<p<\infty$. Then there exists a constant $C_{0}$ depending on $n, q, \Omega$ such that when

$$
\left\|u^{0}\right\|_{\mathcal{L}^{p, q}\left(S_{T}\right)} \leq C_{0},
$$

there exists $T>0$ such that the integral equation:

$$
u+A(u, u)+D(u)=u^{0}
$$

has a solution in $\mathcal{L}^{p, q}\left(S_{T}\right)$.

(ii). Suppose $\frac{n}{p}+\frac{2}{q}<1$ with $n<p<\infty$. Then there exists $T>0$ such that the integral equation (3.11) has a solution in $\mathcal{L}^{p, q}\left(S_{T}\right)$.

PROOF. Let $T(u, v)=A(u, v)+D(u)+u^{0}$. By Theorem 3.1, we have

$$
\|T(u, v)\|_{\mathcal{L}^{p, q}\left(S_{T}\right)} \leq C T^{\frac{1}{2}\left(1-\frac{n}{p}-\frac{2}{q}\right)}\|u\|_{\mathcal{L}^{p, q}\left(S_{T}\right)}\|v\|_{\mathcal{L}^{p, q}\left(S_{T}\right)}+C T\|u\|_{\mathcal{L}^{p, q}\left(S_{T}\right)}^{2}+\left\|u^{0}\right\|_{\mathcal{L}^{p, q}\left(S_{T}\right)} .
$$

Let $c_{1}=C T^{\frac{1}{2}\left(1-\frac{n}{p}-\frac{2}{q}\right)}, c_{2}=C T$ and $\|F\|=\left\|u^{0}\right\|_{\mathcal{C}^{p, q\left(S_{T}\right)}}$. Then if we chose $\left\|u^{0}\right\|_{\mathcal{C}^{p, q\left(S_{T}\right)}}$ and $T$ small enough in case (i) and $T$ small enough in case (ii), conditions in Lemma 3.1 are satisfied. Brown's fixed point theorem implies the existence of solution of integral equation (3.11).

The following theorem assures the uniqueness of the solution of (3.11)

THEOREM 3.3. Suppose $p, q$ satisfy the conditions of Theorem 3.1. Then the solution of (3.11) is unique in the space $\mathcal{L}^{p, q}\left(S_{T}\right)$ for any $T>0$.

PROOF. If $u_{1}, u_{2} \in \mathcal{L}^{p, q}\left(S_{T}\right)$ are two solutions of the integral equation with the same initial data $u_{0}=\left(v_{0}, b_{0}\right)$, then

$$
u_{1}-u_{2}=-\left[A\left(u_{1}, u_{1}-u_{2}\right)+A\left(u_{1}-u_{2}, u_{2}\right)\right]-D\left(u_{1}-u_{2}\right) .
$$

By Theorem 3.1, for $\tau \leq T$,

$$
\left\|u_{1}-u_{2}\right\|_{\mathcal{L}^{p, q}\left(S_{\tau}\right)} \leq \geq C\left(\left\|u_{1}\right\|_{\mathcal{L}^{p, q}\left(S_{\tau}\right)}+\left\|u_{2}\right\|_{\mathcal{L}^{p, q},\left(S_{\tau}\right)}\right)\left\|u_{1}-u_{2}\right\|_{\mathcal{L}^{p, q},\left(S_{\tau}\right)}+C_{\tau}\left\|u_{1}-u_{2}\right\|_{\mathcal{L}^{p, q}\left(S_{\tau}\right)} .
$$

Choose $\tau$ so small that

$$
C\left(\left\|u_{1}\right\|_{\mathcal{L}^{p, q}\left(S_{\tau}\right)}+\left\|u_{2}\right\|_{\mathcal{L}^{p, q}\left(S_{\tau}\right)}\right)+C_{\tau}<1 .
$$

So we see that $u_{1}=u_{2}$ in $S_{\tau}$. Repeating the same procedure, we can cónclude that $u_{1}=u_{2}$ in $S_{2 \tau}$. Continuing repeating the procedure, we can prove finally that $u_{1}=u_{2}$ in $S_{T}$.

Under the conditions of Theorem 3.1, we can easily translate the existence and uniqueness results for the integral equation (2.10) with $F=0$ to respectively existence and uniqueness results for the system (1.1)-(1.5) because of the equivalence result of Theorem 2.1 .

\section{REFERENCES}

[1] HIDE, R., On planetary atmospheres and interiors, Mathematical Problems in the Geophysical Sciences I, W.H. Reid ed., Amer. Math. Soc., Providence, R.I. (1971), p. 229-353.

[2] QU, C., SONG, S. and WANG, P., On the equations for the flows and the magnetic field within the earth, J. Math. Anal. Appl., 187, 3 (1994), 1003-1018.

[3] SERMANGE, M. and TEMAM, R., Some mathematical questions related to the MHD equations, Comm. Pure Appl. Math., 36 (1983), 635-644.

[4] TEMAM, R., Infinite Dimensional Dynamical System in Mechanics and Physics, Springer: Berlin, Heidelberg, New York (1988).

[5] FABES, E.B., JONES, B.F. and RIVIERE, N.M., The initial value problem for the Navier-Stokes equations with data in $L^{p}$, Arch. Rational Mech. Anal., 45 (1972), 222-240.

[6] CALDERÓN, C.P., Existence of weak solutions for the Navier-Stokes equations with initial data in $L^{p}$, Trans. of Amer. Math. Soc., 318, 1 (1990), 179-200. 


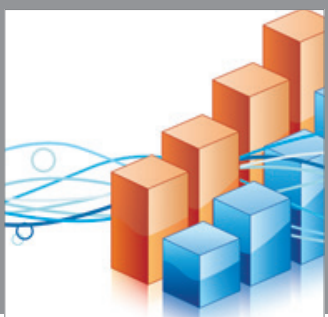

Advances in

Operations Research

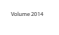

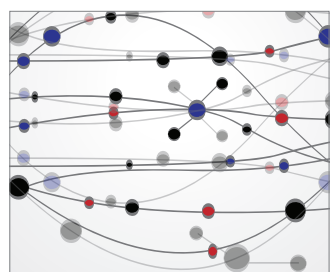

\section{The Scientific} World Journal
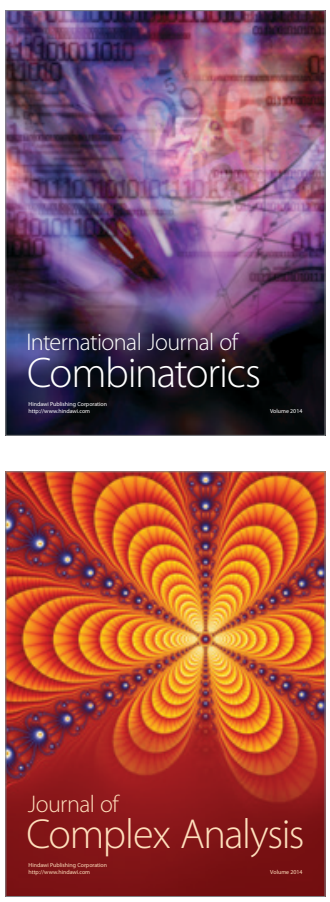

International Journal of

Mathematics and

Mathematical

Sciences
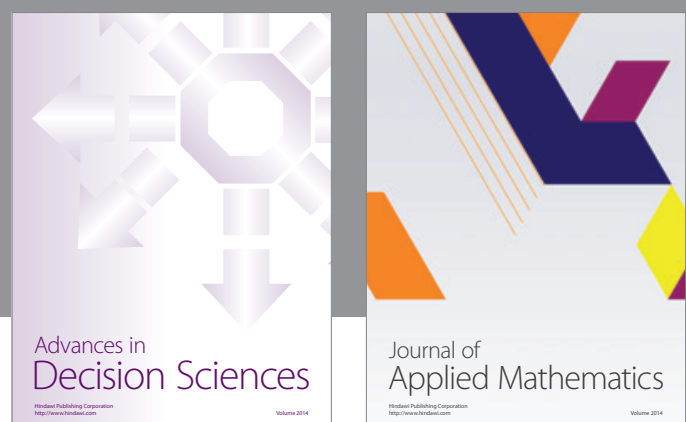

Journal of

Applied Mathematics
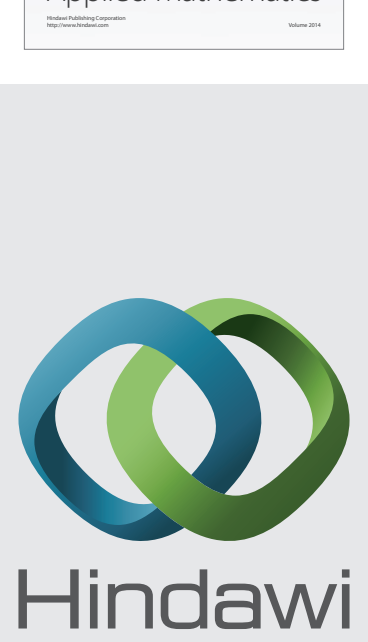

Submit your manuscripts at http://www.hindawi.com
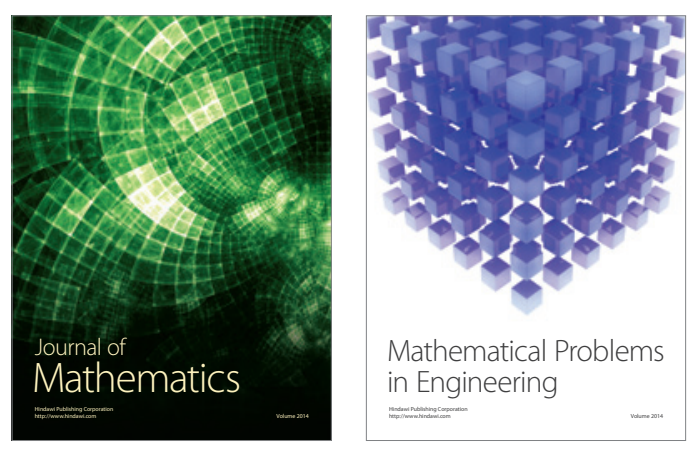

Mathematical Problems in Engineering
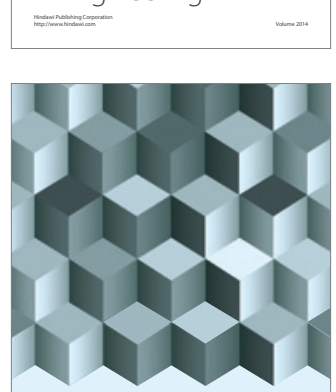

Journal of

Function Spaces
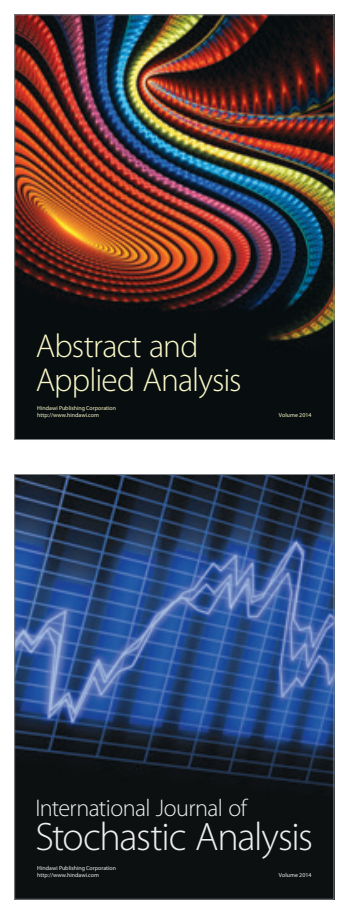

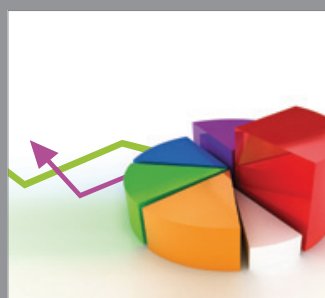

ournal of

Probability and Statistics

Promensencen
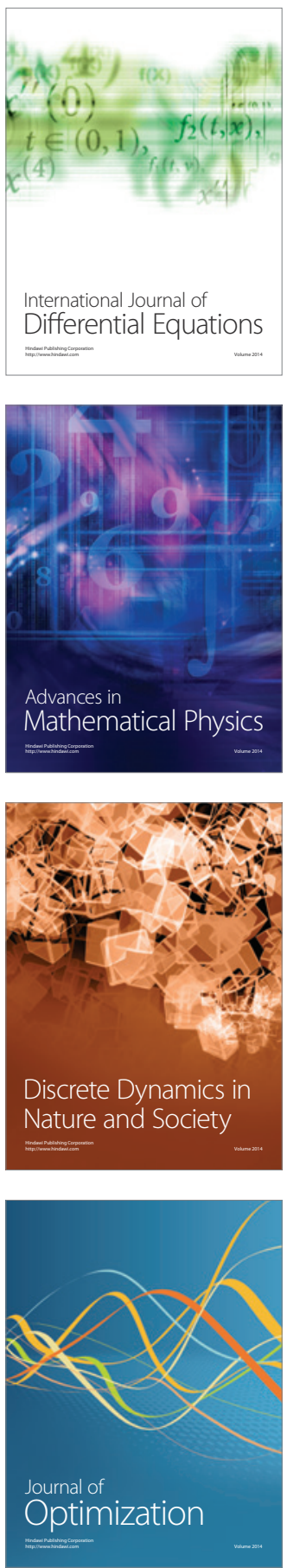